\title{
A Novel Application Potential of GH6 Cellobiohydrolase CtCel6 from Thermophilic Chaetomium thermophilum for Gene Cloning, Heterologous Expression and Biological Characterization
}

\author{
Qinzheng Zhou, Jianchao Jia, Peng Ji and Chao Han* \\ Department of Mycology, Shandong Agricultural University, Taian, Shandong 271018, China \\ *For correspondence: hanch87@163.com
}

\begin{abstract}
Chaetomium thermophilum is a thermophilic fungus expressed a series of glycoside hydrolases. Genome sequence analysis of C. thermophilium revealed that ctcel6 gene encoded a putative cellobiohydrolase which composed of 397 amino acid residues including a predicted signal peptide sequence. Ctcel6 gene was cloned, heterologously expressed in Pichia pastoris and purified by $\mathrm{Ni}^{2+}$ affinity chromatography. Sequence alignment indicated that CtCel6 enzyme belonged to glycoside hydrolase family 6 (GH6) and the molecular mass of purified recombinant enzyme CtCel6 was 42 kDa by SDS-PAGE analysis. Characterization of recombinant $\mathrm{CtCel6}$ exhibited high hydrolysis activity and excellent thermostability. The optimum reaction temperature and $\mathrm{pH}$ was $70^{\circ} \mathrm{C}$ and $\mathrm{pH} 5$, respectively. The bivalent metallic cations $\mathrm{Mg}^{2+}$ and $\mathrm{Ca}^{2+}$ significantly enhanced the activity of CtCel6. The specific activity of CtCel6 enzyme was $1.27 \mathrm{U} / \mathrm{mg}$ and $K_{m}$ value was $0.38 \mathrm{mM}$ on $\beta$-Dglucan. The substrate specificity and hydrolysis products insisted that CtCel6 was an exo-/endo-type cellobiohydrolase. The biochemical properties of recombinant $\mathrm{CtCel6}$ made it potentially effective for bioconversion of biomass and had tremendous potential in industrial applications such as enzyme preparation industry and feed processing industry. (C) 2017 Friends Science Publishers
\end{abstract}

Keyword: Glycoside hydrolase family 6; Cellobiohydrolase; Heterologous expression; Thermostable enzyme; Bioconversion

\section{Introduction}

Lignocellulosic biomass, the most abundantly renewable and available carbohydrate resource on the earth, has been regarded as an available feedstock for biochemical and biotechnological applications to produce biofuels and chemicals (Margeot et al., 2009). Since the global energy crisis and environmental pollution are intensifying, it has attracted extensive attention to the utilization of lignocellulosic biomass. Cellulose is the major composition of lignocellulosic biomass and the bioconversion of cellulose needs a combined effect of three classes of hydrolytic enzymes, including endoglucanases (EC 3.2.1.4), cellobiohyrolases (EC 3.2.1.176; EC 3.2.1.91) and $\beta$ glucosidases (EC 3.2.1.21) (Lynd et al., 2002; Sánchez, 2009). Cellobiohyrolases are a group of glycoside hydrolases, which hydrolyzed oligosaccharides of assorted lengths generated by endoglucanases to cellobiose (Ragauskas et al., 2006). According to the classification of the carbohydrate-active enzymes database (CAZy), cellobiohyrolases are assigned to 5 glycoside hydrolase families: GH5-7, GH9 and GH48 (Cantarel et al., 2009). In particular, the cellobiohydrolases of GH6 are widely believed to act processively from the non-reducing terminal of cellulose chains to generate cellobiose. GH6 family members are mainly produced by bacterial and fungal sources, and the hydrolysis mechanism is inverting. GH6 family includes both endoglucanases and cellobiohydrolases, many GH6 endoglucanases have been reported, such as Thermobifida fusca Cel6A (Ali et al., 2015), Thermobifida halotolerans GH6 endoglucanase (Yin et al., 2015), and Cellulosimicrobium funkei CelL (Kim et al., 2016). However, the study of GH6 cellobiohydrolases has been reported rarely.

Chaetomium thermophilum is a thermophilic fungus living in the high temperature environment up to $60^{\circ} \mathrm{C}$ belonged to the phylum Ascomycota. By now, many glycoside hydrolases have been isolated from $C$. thermophilum, such as a GH55 $\beta$-1,3-glucanase (Papageorgiou and Li, 2015), a $\beta$-glucosidase ( $\mathrm{Xu}$ et al., 2011) and a cellobiohydrolase II (Wang et al., 2013). Generally, C. thermophilum glycoside hydrolases are thermostable and have a high optimal reaction temperature based on the previous researches. Thermostable enzymes have potential advantages in lignocelluloses conversation, on account of effectively improving hydrolysis efficiency and reducing the possible contamination at high temperature in industrial processes (Huy et al., 2016). 
Currently, many microorganism-derived enzymes are produced by fermentation processes in vitro (Villatte et al., 2001). Pichia pastoris, as a convenient production system of yeast that could heterologously express proteins in high amounts, has been wildly used as a heterogeneous expression system (Li et al., 2014; Zhao et al., 2015). To satisfy the strong demand for the thermostable hydrolase production in enzyme preparation industry, heterologous expression using $P$. pastoris has become the primary way which significantly improves the protein expression level (Formighieri and Melis, 2016). However, as a significant part of glycoside hydrolases of thermophilic $C$. thermophilum, there are few reports about the thermostable GH6 cellobiohydrolases from C. thermophilum.

In this study, a novel GH6 cellobiohydrolase gene ctcel6 was first cloned from $C$. thermophilum. The gene ctcel6 was heterologously expressed in P. pastoris system, and the corresponding recombinant enzyme CtCel6 was purified and characterized. The thermostable of CtCel6 was investigated. Moreover, we found it could effectively degrade crystalline cellulose and soluble cellulose at high temperature and the hydrolysis products were mainly cellobiose. The effect of cations and the substrate specific activity of recombinant CtCel6 were also determined. These biochemistry characterizations suggested that recombinant CtCel6 had a great prospect of commercial application in bioconversion of lignocellulosic biomass.

\section{Materials and Methods}

\section{Materials}

Chaetomium thermophilum HSAUP072651 was isolated from bovine feces at Tengchong (Yun'nan, China). The strain was deposited in China General Microbiological Culture Collection Center (CGMCC; Beijing, China) with accession No. 3.17990. Escherichia coli T1 (TransGen, China) was used for nucleotide sequenced. Pichia pastoris strain GS115 (Invitrogen, USA) was used as heterologous expression system. The pMD18-T vector (Takara, China) and the pPIC9K vector (Invitrogen, USA) were used for clone system and expression system, respectively. Primers were synthesized by Sangon Bitech Co. Ltd. (Shanghai, China). RNA extraction kit and reverse transcription kit were purchased from TransGen Company. Sodium carboxymethyl cellulose (CMC-Na), $\beta$-D-glucan, pectin, chitin, xylan, amylose and saccharose were purchased from Sigma-Aldrich. Phosphoric acid swollen cellulose (PASC) was prepared from Avicel according to the method of Wood (1988). Cello-oligosaccharides (Megazyme, Wicklow, Ireland) were used for the hydrolysis products analysis.

\section{Gene Cloning and Sequence Analysis}

After cultured three days with cellulose induced at $50^{\circ} \mathrm{C}$, total RNA of C. thermophilum was isolated from the mycelia as described in the manufacturer's instructions (TransGen, China). Based on the RNA template, reverse transcription was carried out using reverse transcription kit (TransGen, China). The ctcel6 gene was identified from the preview research of genome of C. thermophilum (http://ct.bork.embl.de/) (Amlacher et al., 2011). The gene sequence was amplified using the primers as follows: 5'CCTACGTAGCCCCCAACCCAACCCA-3', and 5'GGCCTAGGTTAGTGGTG

GTGGTGGTGGTGGAACGGAGGGTTGGCA-3'. The designed primers contained a C-terminal 6x His-tagged sequence for the purification of expressed product and the compatible restriction sites of $S n a \mathrm{BI}$ and $A v r \Pi$, respectively. PCR reaction program was as follows: $94^{\circ} \mathrm{C}, 5 \mathrm{~min} ;\left(94^{\circ} \mathrm{C}\right.$, $\left.30 \mathrm{~s} ; 53^{\circ} \mathrm{C}, 30 \mathrm{~s} ; 72^{\circ} \mathrm{C}, 60 \mathrm{~s}\right) \square 30$ cycles; $72^{\circ} \mathrm{C}, 10 \mathrm{~min}$. The amplified product was connected to pMD18-T vector, and the recombinant plasmid was transformed to $E$. coli $\mathrm{T} 1$. The screened positive transformants were gene sequenced to ensure that the cloned gene was correct.

The signal peptide sequence of CtCel6 protein was predicted using the online software SignalP 4.1 (http://www.cbs.dtu.dk/services/SignalP/). The characteristic analysis of CtCel6 amino acid sequence was carried out using the online ProtParam (http://web.expasy.org/protparam/). Multiple sequences alignment was based on ClustalW2 (http://www.simgene.com/ClustalW). Glycosylation sites analysis was performed using NetNGlyc 1.0 (http://www.cbs.dtu.dk/services/NetNGlyc/) for N-linked glycosylation sites and NetOGlyc 4.0 (http://www.cbs.dtu.dk/services/NetOGlyc/) for O-linked glycosylation sites, respectively.

\section{Construction of Expression System}

The cloned gene ctcel6 was digested with SnaBI and $A v r \Pi$, as well as pPIC9K vector. Then, the digested ctcel6 was ligated to pPIC9K vector to produce the secretion expression plasmid $\mathrm{pPIC} 9 \mathrm{~K} /$ ctcel6, which was confirmed by DNA sequencing and restriction analysis. The recombinant plasmid $\mathrm{pPIC} 9 \mathrm{~K} /$ ctcel6 was preserved and prepared for the next step.

\section{Transformation and Expression of the Recombinant Enzyme in Pichia pastoris}

The recombinant expression plasmid pPIC9K/ctcel6 was linearized using the restriction enzyme SacI. After digestion process, pPIC9K/ctcel6 transformed to P. pastoris GS115. Transformants were screened on MD and MM plates at $28^{\circ} \mathrm{C}$ to streak single colony. The multi-copy transformants were picked up from YPD plates with different concentrations of G418 containing 1,2,3 or $4 \mathrm{mg} / \mathrm{mL}$. After incubation $12 \mathrm{~h}$ in YPD liquid medium, genomic DNA extraction was carried out from the selected transformants. A pair of AOX1 sequencing primers (5'- 
GACTGGTTCCAATTGACAAGC-3' and 5'GCAAATGGCATTCTGACATCC-3') was used for PCR amplification with the extracted genomic DNA. The heterogeneous expression program of recombinant CtCel6 was performed in P. pastoris as described by $\mathrm{Li}$ et al. (2009).

\section{Purification and SDS-PAGE Analysis}

The recombinant CtCel6 was purified from the fermentation liquor using $\mathrm{Ni}^{2+}$ affinity chromatography (HisTrap ${ }^{\mathrm{TM}} \mathrm{FF}$ crude, GE Healthcare). The molecular mass of purified recombinant CtCel6 was confirmed by $12 \%(w / v)$ SDSPAGE. The spots of enzyme were stained using Coomassie brilliant blue R-250 and PierceR Glycoprotein Staining Kit (Thermo Scientific, USA), respectively.

\section{Enzyme Assay}

The CtCel6 hydrolysis activity was measured according to the amount of reducing sugar using Nelson-Somogyi method (Miller, 1959). The reaction mixture was composed of $100 \mu \mathrm{L} 0.1 \%(\mathrm{w} / \mathrm{v}) \beta$-D-glucan in $50 \mathrm{mM} \mathrm{pH}$ buffer and $100 \mu \mathrm{L}$ diluted enzyme solution. After reaction incubated for $30 \mathrm{~min}$, the hydrolysis reaction was terminated by adding $200 \mu \mathrm{L}$ 3,5-dinitrosalicylic acid reagent at boiling water within $10 \mathrm{~min}$. After cooled down to ambient temperature, the absorbance was measured at $540 \mathrm{~nm}$. One unit (U) of enzyme activity was defined as the amount of enzyme that catalyzed the liberation of reducing sugar equivalent to $1 \mu \mathrm{moL}$ glucose per minute under assay condition.

\section{Characterization of the Purified CtCel6}

The optimal $\mathrm{pH}$ was determined in $50 \mathrm{mM}$ different buffer solutions ranging from $\mathrm{pH} 3$ to $\mathrm{pH}$ 9. The different $\mathrm{pH}$ buffer solutions were acetate buffer ( $\mathrm{pH} 3-6)$, sodium phosphate (pH 6-8) and Tris- $\mathrm{HCl}(\mathrm{pH} 8-9)$. The optimal temperature was evaluated at $40-90^{\circ} \mathrm{C}$ in $50 \mathrm{mM}$ optimal $\mathrm{pH}$ buffer. Thermal stability was estimated by detecting the residual activity after the enzyme was incubated at 40, 50, $60,70,80$ and $90^{\circ} \mathrm{C}$ for $1 \mathrm{~h}$, respectively. The effect of different metal cations on CtCel6 activity was performed. Substrate specificity was investigated using $\beta$-D-glucan, chitin, pectin, saccharose, xylan, amylose, PASC, CMC-Na, filter paper and wheat straw. $K_{m}$ was determined on $\beta$-D-glucan under the optimum condition and calculated by Michaelis-Menten equation. All assays were performed in triplicate.

The function mechanism of CtCel6 hydrolyzed PASC, CMC-Na and cellooligosaccharides was researched by analyzing the released products. The reaction system on PASC (or CMC-Na) contained of $0.5 \%$ (w/v) PASC (or CMC-Na) and $0.2 \mathrm{mg} / \mathrm{mL}$ CtCel6 in $200 \mu \mathrm{L}$ of reaction mixture at $50^{\circ} \mathrm{C}$ for $3 \mathrm{~h}$ for complete reaction. To detect the hydrolytic products on oligosaccharides, the $200 \mu \mathrm{L}$ reaction mixture containing $0.2 \mathrm{mg} / \mathrm{mL}$ purified CtCel6 and 0.5 $\mathrm{mg} / \mathrm{mL}$ oligosaccharide was incubated at $50^{\circ} \mathrm{C}$ for $3 \mathrm{~h}$ for complete reaction. Hydrolytic products were detected using thin layer chromatography. The reaction products and the oligosaccharides mixture standard (Gentaur, Kampenhout, Belgium) solution were applied onto silica plate (Merck, Germany) and subsequently developed with the developing solvent as ethyl acetate-methanol-water-acetic acid (in the ratio of $4: 2: 1: 0.5, \mathrm{v} / \mathrm{v} / \mathrm{v} / \mathrm{v})$. After completely dried, the developed silica plate was visualized by dipping in a mixed solution containing $2 \%(\mathrm{w} / \mathrm{v}) \mathrm{N}$-phenylaniline, $2 \%(\mathrm{v} / \mathrm{v})$ phenylamine and $85 \%(\mathrm{v} / \mathrm{v})$ phosphoric acid in acetone, followed by heating at $85^{\circ} \mathrm{C}$ for $15 \mathrm{~min}$.

\section{Results}

\section{Sequence Characteristics of CtCel6}

A putative cellobiohydrolase gene ctcel6 (GenBank NO. XM_006694845.1) was isolated using RT-PCR. The gene sequence was $1194 \mathrm{bp}$ and the open reading frame sequence encoded a mature polypeptide, which contained 397 amino acid residues. The calculated molecular mass of the mature polypeptide was $41.8 \mathrm{kDa}$ and the $\mathrm{pH}$ value was 5.7 according to the ProtParam tool. Sequence similarity analysis indicated that CtCel6 belonged to the glycoside hydrolase family 6 compared with other published GH6 family enzymes (Fig. 1). There was a potential signal peptide contained 17 amino acid residues (MKLTTSTLLSLATAALA) in CtCel6 predicted by Signal P 4.1 Server, indicated that it was an exocrine enzyme. Glycosylation sites analysis showed that one N-linked glycosylation sites (N20) and eight O-linked glycosylation sites (T22, T24, T25, S33, S38, T41, T46 and S269) were found in the amino acid sequence according to NetNGlyc 1.0 Server and NetOGlyc 4.0 Server, respectively. It seemed that CtCel6 enzyme could be glycosylated.

\section{Heterologous Expression and Purification}

The expression process of ctcel6 gene in P. pastoris was carried out as described in Pichia Expression Kit (Invitrogen, USA). Transformants were screened and selected the highest producer for further characterization after inducted with methanol. To obtain the purified CtCel6 protein, the screened transformant was cultured under the optimum shake-flask culture condition at $30^{\circ} \mathrm{C}$ for 7 days. The recombinant $\mathrm{CtCel6}$ protein was produced with a $\mathrm{C}$ terminal histidine tag and purified by $\mathrm{Ni}^{2+}$ affinity chromatography. SDS-PAGE analysis indicated that the molecular mass of CtCel6 was $42 \mathrm{kDa}$ closed to the predicted value (Fig. 2). After SDS-PAGE, glycoprotein staining indicated that CtCel6 was a glycoprotein accorded exactly as the prediction (Fig. 2). 


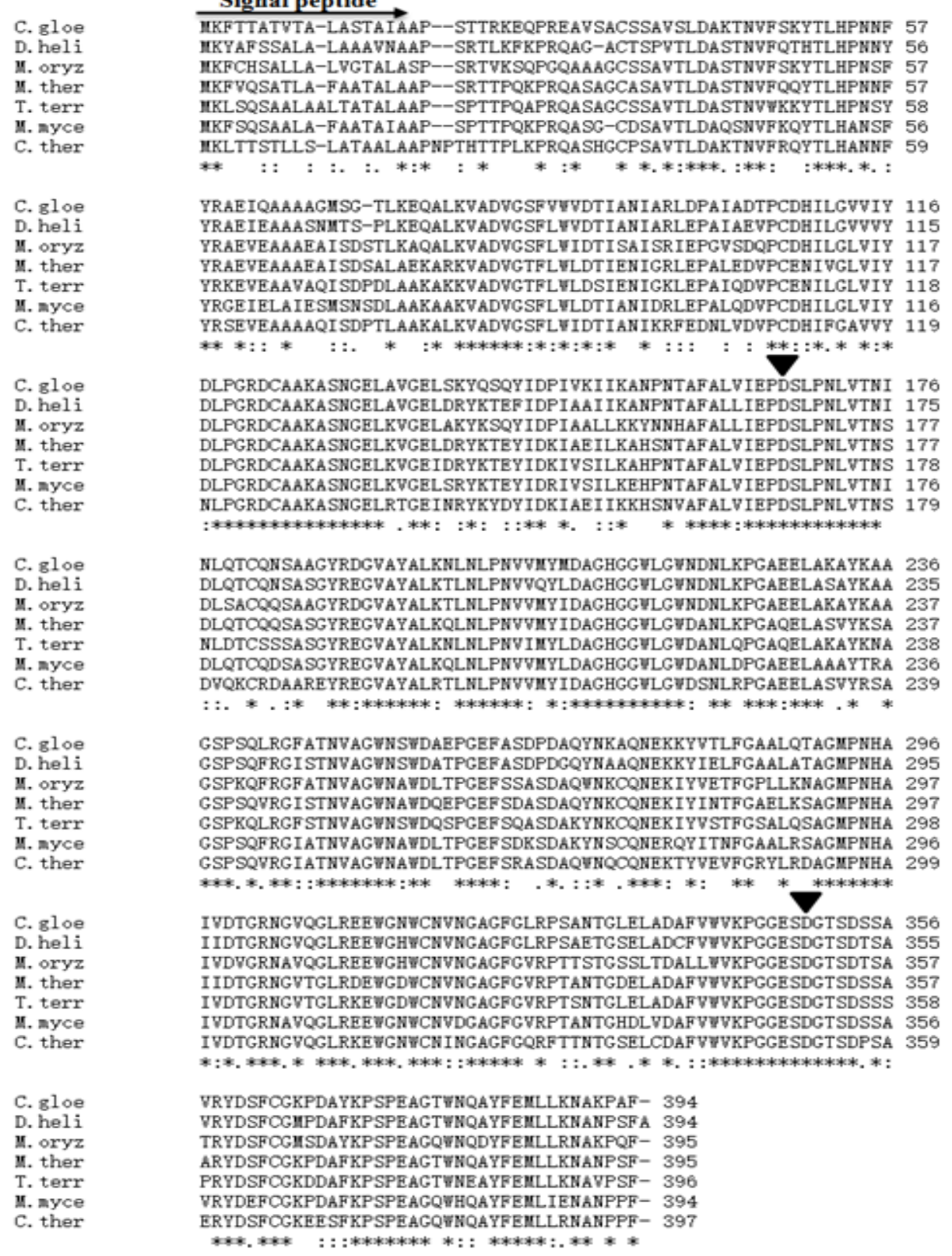

Fig. 1: The sequence alignment of $C$. thermophilium CtCel6 with other GH6 cellobiohydrolases from Colletotrichum gloeosporioides Cg-14 (EQB48544), Diaporthe helianthi (OCW35304), Magnaporthe oryzae 70-15 (XP_003710956), Myceliophthora thermophile ATCC 42464 (XP_003664525), Thielavia terrestris NRRL 8126 (XP_003650908) and Madurella mycetomatis (KXX74427) using ClustalW2. Asterisk indicates the positions which have a single, fully conserved residue. Colon indicates the strongly similar parts among homologous sequences and period means the weakly similar parts among homologous sequences. Two conserved aspartates are noted by closed inverted triangles as the catalytic residues. The potential signal peptide is signed with black arrow

\section{Activity Assay and Characterization of CtCel6}

To characterize the optimal activity condition of CtCel6 enzyme, the optimum temperature and the optimal reaction $\mathrm{pH}$ were measured. The optimum temperature and $\mathrm{pH}$ was $70^{\circ} \mathrm{C}$ (Fig. 3A) and $\mathrm{pH} 5$ (Fig. 3B), respectively.
The thermostability of CtCel6 was also investigated. After incubated 1 hour at different temperatures from $40^{\circ} \mathrm{C}$ to $50^{\circ} \mathrm{C}$, there was little impact on the hydrolysis activity of $\mathrm{CtCel6}$ enzyme. However, after the treatment of $60^{\circ} \mathrm{C}, 70^{\circ} \mathrm{C}$ and $80^{\circ} \mathrm{C}$ for $1 \mathrm{~h}$, the enzyme activity retained $90.5 \%$, $71.3 \%$ and $46.8 \%$, respectively. When pretreated at $90^{\circ} \mathrm{C}$ for 


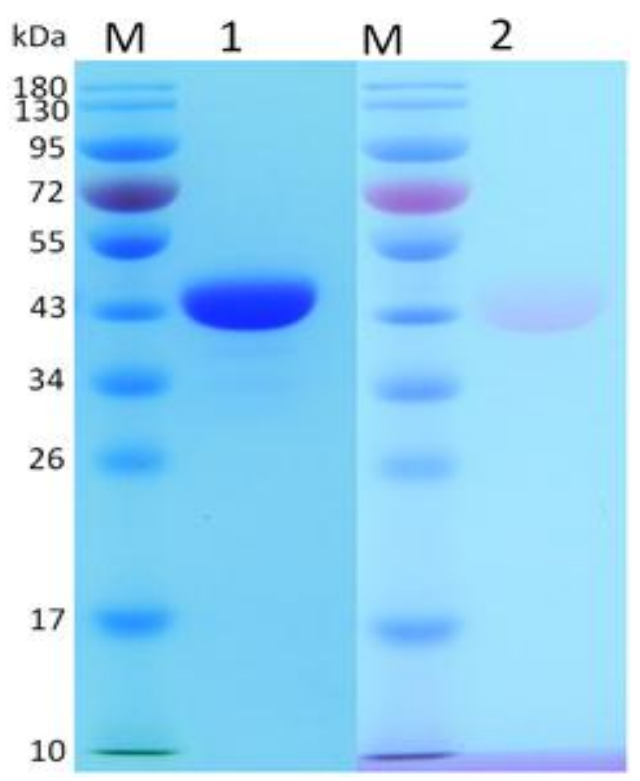

Fig. 2: SDS-PAGE analysis of purified recombinant CtCel6. Lane M, molecular mass marker; lane 1, Coomassie brilliant blue staining; lane 2, carbohydrate staining

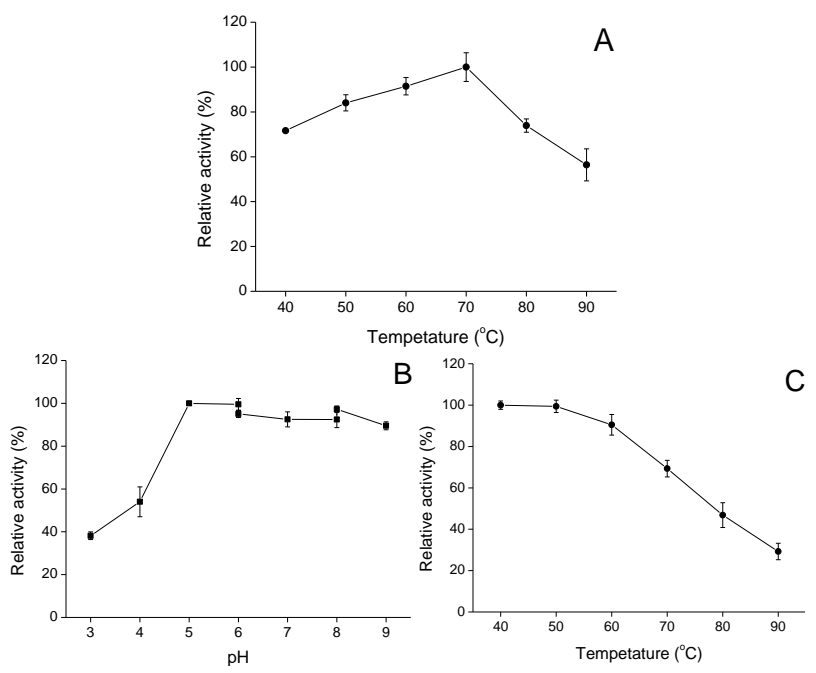

Fig. 3: The optimal temperature (A) and optimal $\mathrm{pH}(\mathrm{B})$ and thermostability $(\mathrm{C})$ of purified recombinant CtCel6. The CtCel6 activity was assayed as described in Materials and Methods. The highest activity was defined as $100 \%$. Values are means $\pm \mathrm{SD}$ of three replications

1 h, only $29.2 \%$ residual activity was detected (Fig. 3C). The effects of various $1 \mathrm{mM}$ metal ions on enzyme activity were also tested. The activity of CtCel6 was significantly enhanced with $\mathrm{Ca}^{2+}$ and $\mathrm{Mg}^{2+}$ by $151.6 \%$ and $149.9 \%$, respectively. In contrast, $\mathrm{Hg}^{2+}, \mathrm{Mn}^{2+}, \mathrm{Cu}^{2+}$ and $\mathrm{Fe}^{3+}$ reduced the enzyme activity at different degrees. Monovalent cation $\mathrm{K}^{+}$and $\mathrm{Na}^{+}$were detected no obvious effect, as well as $\mathrm{Zn}^{2+}$
Table 1: Effect of cations on the activity of purified CtCel6

\begin{tabular}{ll}
\hline Metal ions & Relative activity (\%) \\
\hline Control & $100.0 \pm 2.5$ \\
$\mathrm{Na}^{+}$ & $100.5 \pm 5.4$ \\
$\mathrm{~K}^{+}$ & $96.1 \pm 4.2$ \\
$\mathrm{Zn}^{2+}$ & $100.6 \pm 11.2$ \\
$\mathrm{Mg}^{2+}$ & $149.9 \pm 4.8$ \\
$\mathrm{Hg}^{2+}$ & $31.9 \pm 3.6$ \\
$\mathrm{Ca}^{2+}$ & $151.6 \pm 2.9$ \\
$\mathrm{Mn}^{2+}$ & $39.9 \pm 5.6$ \\
$\mathrm{Cu}^{2+}$ & $38.4 \pm 4.5$ \\
$\mathrm{Fe}^{3+}$ & $65.7 \pm 6.7$ \\
$\mathrm{Al}^{3+}$ & $103.3 \pm 3.9$ \\
\hline Control is measured without metal ions. The final concentration of each \\
the metal ions is 1 mM. The activity was determined as described in \\
Materials and Methods. Values are means \pm SD of three replications
\end{tabular}

Table 2: Substrate specific activity of CtCel6

\begin{tabular}{ll}
\hline Substrate & Specific activity $(\mathrm{U} / \mathrm{mg})$ \\
\hline$\beta$-D-Glucan & $1.27 \pm 0.10$ \\
Pectin & $1.90 \pm 0.07$ \\
PASC & $0.95 \pm 0.06$ \\
Filter paper & $0.39 \pm 0.01$ \\
Wheat straw & $0.37 \pm 0.01$ \\
CMC-Na & $0.11 \pm 0.03$ \\
Chitin & ND \\
Saccharose & ND \\
Xylan & ND \\
Amylose & ND \\
\hline
\end{tabular}

ND means not detected

The concentration of $\beta$-D-glucan is $0.1 \%(\mathrm{w} / \mathrm{v})$, as well as pectin. PASC, CMC-Na, saccharose, xylan and amylose are used as $0.5 \%(\mathrm{w} / \mathrm{v})$. Chitin, filter paper and wheat straw are $20 \mathrm{mg}$ in each reaction. PASC was prepared from Avicel as described in Materials and Methods. Wheat straw is not pretreatment. Values are means $\pm \mathrm{SD}$ of three replications

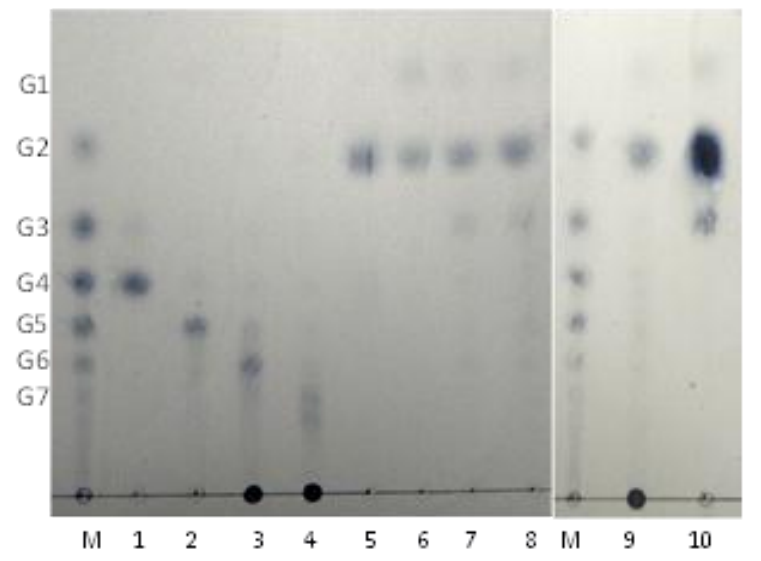

Fig. 4: Analysis of hydrolytic products by thin layer chromatography. Assay methods are described in the part of Materials and Methods. Lane M, the oligosaccharides mixture standard; lane 1, cellotetraose; lane 2, cellopentaose; lane 3, cellohexaose; lane4, celloheptaose; lane 5 to lane 8 mean the hydrolytic products on cellotetraose, cellopentaose, cellohexaose and celloheptaose, respectively. Lane 9, the hydrolytic products on CMC-Na; Lane 10, the hydrolytic products on PASC. G1to G7 means the degree of polymerization of cellulose 
and $\mathrm{Al}^{3+}$ (Table 1). The observation of substrate specificity showed that CtCel6 displayed high activity towards $\beta$-Dglucan with $1.27 \mathrm{U} / \mathrm{mg}$, lower activity on PASC with 0.95 $\mathrm{U} / \mathrm{mg}$ and slight activity on CMC-Na. Almost no detectable activity was found on chitin, saccharose, xylan and amylose (Table 2). The corresponding kinetic parameter $K_{m}$ was 0.38 $\mathrm{mM}$ on $\beta$-D-glucan.

The hydrolysis products were mainly cellobiose released from PASC, and lower amounts of cellotriose and glucose were liberated. The similar result was detected on $\mathrm{CMC}-\mathrm{Na}$, but the amount of reaction products relatively lower than those on PASC. After reacted at $50^{\circ} \mathrm{C}$ for $3 \mathrm{~h}$, CtCel6 hydrolyzed cello-oligosaccharides completely and each substrate disappeared from the reaction. The sole product on cellotetraose was cellobiose. The end products on cellopentaose were mainly cellobiose and low amounts of glucose. CtCel6 converted cellohexaose and celloheptaose into cellotriose, cellobiose and glucose under react condition (Fig. 4).

\section{Discussion}

By now, many GH6 family enzymes have been isolated and experimentally characterized from multiplying fungi (Zhang et al., 2000; Tao et al., 2014; Kim et al., 2016). However, as an important part of cellobiohydrolases, only a few reports on the GH6 cellobiohydrolases from thermophilic fungi (Thompson et al., 2012). Herein, a new cellobiohydrolase gene ctcel 6 was first cloned from thermophilic $C$. thermophilum and heterologous expressed in $P$. pastoris system. Blastp analyzed the amino acid sequence similarity of CtCel6 protein, the result revealed a relatively high degree of identity with other glycoside hydrolase family 6 members. CtCel6 shared 78\% identity with Myceliophthora thermophile GH6 protein (XP_003664525), 73\% identity with Thielavia terrestris GH6 protein (XP_003650908) and 72\% identity with Magnaporthe oryzae exoglucanase-6A (XP_003710956). Some aromatic amino acid residues were found in CtCel6, which were supposed to be cellulose substrate-binding sites of GHs (Takashima et al., 2007). Two conserved Asp residues D170 and D352 were observed (Fig. 1), suggesting that they are likely to be involved in catalytic activity as proton donor and nucleophile. Moreover, SDS-PAGE analysis showed CtCel6 was a glycoprotein (Fig. 2). Glycosylation, as a common post-translational modification, has important function in structure and function of enzymes. Generally, sugar residues are linked to Ser, Thr, Hyl and Asp residues in the way of O-linked and N-linked. Recent evidences in the literature suggested that the enzymatic glycosylation might be concerned with stability (Chen et al., 2014; Tao et al., 2014).

The result showed that CtCel6 has high thermostability and a high temperature activity (Fig. $3 \mathrm{~A}, \mathrm{C})$, similar to other enzymes of thermophilic fungi
(Li et al., 2011; Shi et al., 2014). Thermostable CtCel6 should play a major role to adapt the high temperature environment for $C$. thermophilum. These properties indicated that thermostable cellobiohydrolase CtCel6 has practical value and great commercial application potential. Generally, in most operational situations, the optimal temperature of enzymatic action is $40-60^{\circ} \mathrm{C}$, it is considered that high temperature reaction activity and thermostable enzyme is favorable (Aditya et al., 2014; Huy et al., 2016). In addition, the purified recombinant $\mathrm{CtCel} 6$ exhibited excellent activity in a range of $\mathrm{pH} 5$ to $\mathrm{pH} 9$ with the maximum hydrolysis activity at $\mathrm{pH} 5$ as showen in Fig. 3B, which was similar to cellobiohydrolases from other thermophilic fungi, such as Fusarium chlamydosporum, Thermoascus aurantiacus and Thielavia terrestris. (Qin et al., 2010; Hong et al., 2003; Woon et al., 2016). The bivalent metallic cations $\mathrm{Mg}^{2+}$ and $\mathrm{Ca}^{2+}$ significantly enhanced the activity of $\mathrm{CtCel6}$, while the monovalent cations $\mathrm{K}^{+}$and $\mathrm{Na}^{+}$were not detected with obvious effect (Table 1). Similar conclusion was reported on GH9 endoglucanase CenC from Clostridium thermocellum and $\beta$ glucosidase Bgl4 from Penicillium funiculosum (Haq et al., 2015; Ramani et al., 2015), owing to bivalent metallic cations could increase the stability and cause conformational changes of catalytic center of cellulose. The observation of substrate specificity showed that $\mathrm{CtCel6}$ displayed high activity towards $\beta$-D-glucan with $1.27 \mathrm{U} / \mathrm{mg}$, which was higher than two cellobiohydrolase (CBHI, CBHII) from Trichoderma viride (Song et al., 2010), but lower than cellobiohydrolase PcCel6A from Paenibacillus curdlanolyticus (Baramee et al., 2016). PASC could also be hydrolyzed by CtCel6 as showed in Table 2. According to CAZy enzyme classification, cellobiohydrolases act on the terminal of cellulose chains to remove cellobiose, while the role of endoglucanases is to produce a series of compounded cellooligosaccharides. During the hydrolysis reaction for $3 \mathrm{~h}$, the major product was cellobioses on each cellooligosaccharides by thin layer chromatography analysis. This result indicated that $\mathrm{CtCel6}$ was a cellobiohydrolase, instead of an endoglucanase (Fig. 4). The hydrolyzed products on PASC and CMC-Na were mainly cellobiose (Fig. 4). As previously reported, some cellobiohydrolases of GH6 were detected to hydrolyze $\mathrm{CMC}-\mathrm{Na}$, as well as PASC, which were defined as the exo/endo-type cellobiohydrolases (Wang et al., 2013; Baramee et al., 2016). In exo-/endo-type cellobiohydrolases, the flexible tunnel-like active sites are the reason that causing exo-type action connected with endo-type action. Nevertheless, true exo-type cellobiohydrolases appear to hydrolyse crystalline cellulose, but $\mathrm{CMC}-\mathrm{Na}$ is not their substrate. Consequently, the analysis of substrate specificity and hydrolysis products highlighted that CtCel6 was an exo-/endo-type cellobiohydrolase (Table 2; Fig. 4). The majority of crystalline cellulose degradation is performed by exo-type cellobiohydrolases, while soluble cellulose could be 
hydrolyzed by endo-type cellobiohydrolase. As a result, exo-/endo-type cellobiohydrolases are necessary for lignocellulosic biomass biodegradation in industry, especially the thermostable cellobiohydrolases.

\section{Conclusion}

We cloned a new GH6 cellobiohydrolase gene ctcel6 from thermophilic $C$. thermophilum and heterologously expressed in P. pastoris system. The corresponding recombinant protein CtCel6 was purified and detected its characteristics. These results demonstrated that the recombinant CtCel6 had high hydrolysis activity and excellent thermostability at elevated temperature. These properties are considered essential conditions for enzymic preparation which make CtCel6 act as an interesting potential candidate for biochemical and bioindustrial applications.

\section{Acknowledgments}

This research was supported by National Key Technology R\&D Program of China funded by Ministry of Science and Technology (Grant NO. 2015BAD15B05). Authors thank Prof. Duochuan Li from Shandong Agricultural University for his kind help with the genome analysis.

\section{References}

Aditya, B., M.B. Kenneth, U. Nirmal, B. Venkatesh and K.S. Rajesh, 2014. Novel thermostable endo-xylanase cloned and expressed from bacterium Geobacillus sp. WSUCF1. Bioresour. Technol., 165: 314 318

Ali, I., R. Asghar, S. Ahmed, M. Sajjad, M. Tariq and M. Waheed Akhtar, 2015. mRNA secondary structure engineering of Thermobifida fusca endoglucanase (Cel6A) for enhanced expression in Escherichia coli. World. J. Microbiol. Biotechnol., 31: 499-506

Amlacher, S., P. Sarges, D. Flemming, V. van Noort, R. Kunze, D.P. Devos, M. Arumugam, P. Bork and E. Hurt, 2011. Insight into structure and assembly of the nuclear pore complex by utilizing the genome of a eukaryotic thermophile. Cell, 146: 277-289

Baramee, S., T. Teeravivattanakit, P. Phitsuwan, R. Waeonukul, P. Pason, C. Tachaapaikoon, A. Kosugi, K. Sakka and K. Ratanakhanokchai, 2016. A novel GH6 cellobiohydrolase from Paenibacillus curdlanolyticus B-6 and its synergistic action on cellulose degradation. Appl. Microbiol. Biotechnol., DOI: 10.1007/s00253016-7895-8

Cantarel, B.L., P.M. Coutinho, C. Rancurel, T. Bernard, V. Lombard and B. Henrissat, 2009. The Carbohydrate-Active EnZymes database (CAZy): An expert resource for glycogenomics. Nucleic Acids. Res., 37: D233-D238

Chen, X.L., T. Shi, J. Yang, W. Shi, X.S. Gao, D. Chen, X.W. Xu, J.R. Xu, N.J. Talbot and Y.L. Peng, 2014. N-glycosylation of effector proteins by an $\alpha-1,3-$ mannosyltransferase is required for the rice blast fungus to evade host innate immunity. Plant Cell, 26: 1360-1376

Formighieri, C. and A. Melis, 2016. Sustainable heterologous production of terpene hydrocarbons in cyanobacteria. Photosynth. Res. Dec., 130: 123-135

Haq, I., F. Akram, M.A. Khan, Z. Hussain, A. Nawaz, K. Iqbal and A.J. Shah, 2015. CenC, a multidomain thermostable GH9 processive endoglucanase from Clostridium thermocellum: cloning, characterization and saccharification studies. World. J. Microbiol. Biotechnol., 31: 1699-1710
Hong, J., H. Tamaki, K. Yamamoto and H. Kumagai, 2003. Cloning of a gene encoding thermostable cellobiohydrolase from Thermoascus aurantiacus and its expression in yeast. Appl. Microbiol. Biotechnol., 63: $42-50$

Huy, N.D., C.L. Nguyen, H.S. Park, N.H. Loc, M.S. Choi, D.H. Kim, J.W. Seo and S.M. Park, 2016. Characterization of a novel manganese dependent endoglucanase belongs in $\mathrm{GH}$ family 5 from Phanerochaete chrysosporium. J. Biosci. Bioeng., 121: 154-159

Kim, do Y., M.J. Lee, H.Y. Cho, J.S. Lee, M.H. Lee, C.W. Chung, D.H. Shin, Y.H. Rhee, K.H. Son and H.Y. Park, 2016. Genetic and functional characterization of an extracellular modular GH6 endo- $\beta$ 1,4-glucanase from an earthworm symbiont, Cellulosimicrobium funkei HY-13. Antonie. Van. Leeuwenhoek., 109: 1-12

Li, D.C., A.N. Li and A.C. Papageorgiou, 2011. Cellulases from thermophilic fungi: recent insights and biotechnological potential. Enzyme. Res., 2011: 308-730

Li, H.B., Y.H. C, L.W. Zhang, X.Y. Hua, H. Xue, H.J. Yue, D. Ming, B.F. Rong and S. Zhang, 2014. Heterologous expression and purification of Zea mays transglutaminase in Pichia pastoris. Food. Sci. Biotechnol., 23: 1507-1513

Li, Y.L., H. Li, A.N. Li and D.C. Li, 2009. Cloning of a gene encoding thermostable cellobiohydrolase from the thermophilic fungus Chaetomium thermophilum and its expression in Pichia pastoris. $J$. Appl. Microbiol., 106: 1867-1875

Lynd, L.R., P.J. Weimer, W.H. van Zyl and I.S. Pretorius, 2002. Microbial cellulose utilization: fundamentals and biotechnology. Microbiol. Mol. Biol. Rev., 66: 506-577

Margeot, A., B. Hahn-Hagerdal, M. Edlund, R. Slade and F. Monot, 2009. New improvements for lignocellulosic ethanol. Curr. Opin. Biotechnol., 20: 372-380

Miller, G.L., 1959. Use of dinitrosalicylic reagent for determination of reducing sugar. Anal. Chem., 31: 426-428

Papageorgiou, A.C. and D. Li, 2015. Expression, purification and crystallization of a family $55 \beta$-1,3-glucanase from Chaetomium thermophilum. Acta. Cryst. F., 71: 680-683

Qin, Y.L., H.Y. He, N. Li, M. Ling and Z.Q. Liang, 2010. Isolation and characterization of a thermostable cellulase-producing Fusarium chlamydosporum. World. J. Microbiol. Biotechnol., 26: 1991-1997

Ragauskas, A.J., C.K. Williams, B.H. Davison, G. Britovsek, J. Cairney, C.A. Eckert, W.J. Jr. Frederick, J.P. Hallett, D.J. Leak, C.L. Liotta, J.R. Mielenz, R. Murphy, R. Templer and T. Tschaplinski, 2006. The path forward for biofuels and biomaterials. Science, 311: 484-489

Ramani, G., B. Meera, C. Vanitha, J. Rajendhran and P. Gunasekaran, 2015 Molecular cloning and expression of thermostable glucose-tolerant $\beta$ glucosidase of Penicillium funiculosum NCL1 in Pichia pastoris and its characterization. J. Ind. Microbiol. Biotechnol. Apr., 42: 553-565

Sánchez, C., 2009. Lignocellulosic residues: biodegradation and bioconversion by fungi. Biotechnol. Adv., 27: 185-194

Shi, H., Y. Zhang, H. Zhong, Y.J. Huang, X. Li and F. Wang, 2014. Cloning, over-expression and characterization of a thermotolerant xylanase from Thermotoga thermarum. Biotechnol. Lett., 36: 587593

Song, J.Z., B.D. Liu, Z.H Liu and Q. Yang, 2010. Cloning of two cellobiohydrolase genes from Trichoderma viride and heterogenous expression in yeast Saccharomyces cerevisiae. Mol. Biol. Rep., 37: $2135-2140$

Takashima, S., M. Ohono, M. Hidaka, A. Nakamura, H. Masaki and T. Uozumi, 2007. Correlation between cellulose binding and activity of cellulose-binding domain mutants of Humicola grisea cellobiohydrolase. FEBS. Lett., 581: 5891-5896

Tao, L, Z.M. Zhang and C. Zhang, 2014. Glycosyl rotation and distortion by key residues in endocellulase Cel6A from Theromobifida fusca. Glycobiology, 24: 247-251

Thompson, A.J., T. Heu, T. Shaghasi, R. Benyamino, A. Jones, E.P. Friis, K.S. Wilson and G.J. Davies, 2012. Structure of the catalytic core module of the Chaetomium thermophilum family GH6 cellobiohydrolase Cel6A. Acta. Crystallogr. D Biol. Crystallogr., 68: 875-882

Villatte, F., A. Hussein, T. Bachmann and R. Schmid, 2001. Expression level of heterologous proteins in Pichia pastoris is influenced by flask 
design. Appl. Microbiol. Biotechnol., 55: 463-465

Wang, H.C., Y.C. Chen, C.T. Huang and R.S. Hseu, 2013. Cloning and characterization of a thermostable and $\mathrm{pH}$-stable cellobiohydrolase from Neocallimastix patriciarum J11. Protein. Exp. Purif., 90: 153159

Wood, T.M., 1988. Preparation of crystalline, amorphous, and dyed cellulase substrates. Methods. Enzymol., 160: 19-25

Woon, J.S., M.M. Mackeen, A.H. Sudin, N.M. Mahadi, R.M. Illias, A.M. Murad and F.D. Bakar, 2016. Erratum to: Production of an oligosaccharide-specific cellobiohydrolase from the thermophilic fungus Thielavia terrestris. Biotechnol. Lett., 38: 825-832

$\mathrm{Xu}, \mathrm{R}$., F. Teng, C. Zhang and D. Li, 2011. Cloning of a gene encoding $\beta$ glucosidase from Chaetomium thermophilum $\mathrm{CT} 2$ and its expression in Pichia pastoris. J. Mol. Microbiol. Biotechnol., 20: 16-23
Yin, Y.R., F. Zhang, Q.W. Hu, W.D. Xian, W.N. Hozzein, E.M. Zhou, H. MingandG.X.Nie, 2015. Heterologous expression and characterization of a novel halotolerant, thermostable, and alkali-stable GH6 endoglucanase from Thermobifida halotolerans. Biotechnol. Lett., 37: $857-862$

Zhang, S., B.K. Barr and D.B. Wilson, 2000. Effects of noncatalytic residue mutations on substrate specificity and ligand binding of Thermobifida fusca endocellulase Cel6A. Eur. J. Biochem., 267: 244-252

Zhao, J.Q., C. Guo, C.G. Tian and Y.H. Ma, 2015. Heterologous expression and characterization of a GH3 $\beta$-glucosidase from thermophilic fungi Myceliophthora thermophila in Pichia pastoris. Appl. Biochem. Biotechnol., 177: 511-527

(Received 28 November 2016; Accepted 4 February 2017) 